\title{
The SS433 jet from subparsec to parsec scales (Corrigendum)
}

\author{
Remi Monceau-Baroux ${ }^{1}$, Oliver Porth ${ }^{2}$, Zakaria Meliani $^{3}$, and Rony Keppens ${ }^{1}$ \\ ${ }^{1}$ Centre for mathematical Plasma Astrophysics, Department of Mathematics, KU Leuven, Celestijnenlaan 200B, 3001 Heverlee, \\ Belgium \\ e-mail: remi.monceaubaroux@gmail.com \\ 2 Institute for Theoretical Physics, Frankfurt am Main, 60438 Germany \\ 3 LUTh, Observatoire de Paris, France
}

A\&A 574, A143 (2015), DOI: 10.1051/0004-6361/201425015

Key words. hydrodynamics - relativistic processes - ISM: jets and outflows - X-rays: binaries - errata, addenda

Our paper Monceau-Baroux et al. (2015) presented numerical simulations of the precessing SS433 jet up to parsec scales, and its main finding was a dynamical recollimation effect: the jet transits from a winding helix to a more hollow straight jet. We argued that this transition occurred at 0.068 parsec, and gave a physical argument based on ram pressure that balances the pressure of the interstellar medium (ISM). This writes as $\rho_{0} \gamma_{0}^{2} v_{0}^{2} d_{0}^{2} / d_{1}^{2}=P_{\text {ISM }}$ (our Eq. (2)), where the $\rho_{0} d_{0}^{2} / d_{1}^{2}$ is intended to estimate the density decrease in the jet beam at a distance $d_{1}$, while the jet is injected at $d_{0}$. The factor $v_{0}^{2}$ (and its Lorentz factor $\gamma_{0}$, relatively small in the case of SS433) quantifies the squared beam velocity. Given the constant ISM pressure $\left(7.5 \times 10^{-6} \mathrm{~g} \mathrm{~cm}^{-1} \mathrm{~s}^{-2}\right)$, the distance $d_{0}=0.008 \mathrm{pc}$, and typical density/velocity values, this can quantify the distance $d_{1}$ where effects caused by the ISM pressure occur. Unfortunately, the estimate $d_{1} \approx 8.5 d_{0}$ (i.e. $0.068 \mathrm{pc}$ ) we quoted in Eq. (3) used the ISM density for $\rho_{0}$ (namely $\rho_{\text {ISM }}=8.3 \times 10^{-24} \mathrm{~g} \mathrm{~cm}^{-3}$ ), together with a beam speed of $v_{\mathrm{b}}=0.26 c$. A corrected estimate with the actual beam density adopted in our study $\left(\rho_{b}=2.58 \times 10^{-22} \mathrm{~g} \mathrm{~cm}^{-3}\right.$ at injection) instead gives $d_{1} \approx 47.6 d_{0}$, which is factor of 5.6 larger. This value clearly overestimates the distance where recollimation was found to occur (see Figs. 4, 6, or 8 in our paper). An improved estimate can be obtained by acknowledging that the ram pressure acts directional, such that a projection using the fixed precession angle $\theta_{\text {prec }}=20^{\circ}$ enters the left-hand side. If we quantify where the beam flow component away from the precession axis (i.e., adopt $v_{0}=v_{\mathrm{b}} \sin \left(\theta_{\text {prec }}\right)$ ) matches the ISM pressure in ram pressure, we obtain $d_{1} \approx 15.8 d_{0}$, or a distance of 0.126 parsec. This is in better agreement with the observed recollimation. Deviations from this simple estimate can be understood from the fact that the full 3D jet-ISM interaction rather progresses with a decreased head speed of $0.185 c$ due to deceleration (Monceau-Baroux et al. 2014), which further reduces the recollimation distance. Furthermore, the jet propagation itself disturbs and modifies the neighboring density-pressure ISM conditions, and may in particular modify the pressure distribution interior to the helical jet path. This will occur on a timescale associated with the jet thermal expansion toward the axis, and this effect can similarly lower the obtained distance estimate. We further note that earlier models (Eichler 1983) predicted a complete refocusing of a hollow, conical, axisymmetric jet (with zero pressure within the hollow cone) over a distance estimated as $3\left(L / \pi v_{\mathrm{b}} P_{\mathrm{ISM}}\right)^{1 / 2}$, with $L$ the jet power. This model balances the pressure on the jet with the centrifugal force felt by a jet parcel along its curved trajectory in the plane containing the jet axis. As the SS433 kinetic luminosity $L=10^{39} \mathrm{erg} \mathrm{s}^{-1}$, this estimate leads to $0.07 \mathrm{pc}$, implying that deflection effects would occur at half this distance, or at $0.035 \mathrm{pc}$. Clearly, this underestimates where our simulations find the deflection. This highlights the role of accounting for the full 3D helical nature of the jet flow, and for the finite pressure effects in the jet surroundings.

Acknowledgements. We thank Michael Bowler for pointing out this error, and bringing up relevant references and discussions.

\section{References}

Eichler, D. 1983, ApJ, 272, 48

Monceau-Baroux, R., Porth, O., Meliani, Z., \& Keppens, R. 2014, A\&A, 561, A30

Monceau-Baroux, R., Porth, O., Meliani, Z., \& Keppens, R. 2015, A\&A, 574, A143 